\title{
Discrete Schrödinger equations in the nonperiodic and superlinear cases: homoclinic solutions
}

\author{
Liqian Jia' ${ }^{1}$ Jun Chen² and Guanwei Chen ${ }^{1 *}$
}

\author{
"Correspondence: \\ guanweic@163.com \\ ${ }^{1}$ School of Mathematical Sciences, \\ University of Jinan, Jinan, Shandong \\ Province 250022, P.R. China \\ Full list of author information is \\ available at the end of the article
}

\begin{abstract}
Using variational methods, we study the existence and multiplicity of homoclinic solutions for a class of discrete Schrödinger equations in infinite $m$-dimensional lattices with nonlinearities being superlinear at infinity. Our results generalize some existing results in the literature by using some weaker conditions.
\end{abstract}

MSC: 35Q51;35Q55; 39A12; 39A70

Keywords: discrete nonlinear Schrödinger equations; variational methods; Superlinear; homoclinic solutions

\section{Introduction and main results}

The discrete nonlinear Schrödinger equation is a very important discrete model, which has many important applications in many fields, such as nonlinear optics [1], biomolecular chains [2], Bose-Einstein condensates [3], and so on.

In general, discrete nonlinear Schrödinger equation can be divided into two different cases, the periodic and nonperiodic cases. So far, most results are all about the periodic cases, such as [4-16] and so on. Only a few results are about the nonperiodic cases, such as [17-28]; in paticular, the papers $[17,21,22,24,27]$ are only about the case of onedimensional lattice $(n \in \mathbb{Z})$.

Inspired by the papers mentioned, we study homoclinic solutions $\left(\lim _{|n|=\left|n_{1}\right|+\left|n_{2}\right|+\cdots+\left|n_{m}\right| \rightarrow \infty} u_{n}=0\right)$ of the following nonperiodic discrete nonlinear equation:

$$
-\Delta u_{n}+v_{n} u_{n}-\omega u_{n}=f_{n}\left(u_{n}\right), \quad n \in \mathbb{Z}^{m},
$$

where

$$
\begin{aligned}
\Delta u_{n}= & u_{\left(n_{1}+1, n_{2}, \ldots, n_{m}\right)}+u_{\left(n_{1}, n_{2}+1, \ldots, n_{m}\right)}+\cdots+u_{\left(n_{1}, n_{2}, \ldots, n_{m}+1\right)}-2 m u_{\left(n_{1}, n_{2}, \ldots, n_{m}\right)} \\
& +u_{\left(n_{1}-1, n_{2}, \ldots, n_{m}\right)}+u_{\left(n_{1}, n_{2}-1, \ldots, n_{m}\right)}+u_{\left(n_{1}, n_{2}, \ldots, n_{m}-1\right)}
\end{aligned}
$$

is the discrete Laplace operator in the $m$-dimensional space, $\omega \in \mathbb{R}, V=\left\{v_{n}\right\}_{n \in \mathbb{Z}^{m}}$ and $\left\{u_{n}\right\}_{n \in \mathbb{Z}^{m}}$ are sequences of real numbers, and the nonlinearities $f_{n}$ satisfy

$$
f_{n}\left(e^{i \omega} s\right)=e^{i \omega} f_{n}(s), \quad \forall \omega \in \mathbb{R}, \forall(n, s) \in \mathbb{Z}^{m} \times \mathbb{R} .
$$

(c) The Author(s) 2017. This article is distributed under the terms of the Creative Commons Attribution 4.0 International License (http://creativecommons.org/licenses/by/4.0/), which permits unrestricted use, distribution, and reproduction in any medium, provided you give appropriate credit to the original author(s) and the source, provide a link to the Creative Commons license, and indicate if changes were made. 
Problem (1.1) comes from the study of standing waves for the discrete nonlinear Schrödinger equation

$$
i \dot{\psi}_{n}=-\Delta \psi_{n}+v_{n} \psi_{n}-f_{n}\left(\psi_{n}\right), \quad n \in \mathbb{Z}^{m}
$$

Clearly, (1.2) becomes (1.1) by the definition of standing waves $\left(\psi_{n}=u_{n} e^{-i \omega t}\right.$ with $\lim _{|n| \rightarrow \infty} u_{n}=0$ ). Therefore, the problem on the existence of standing waves of (1.2) reduces to that on the existence of homoclinic solutions of (1.1).

We use some suitable assumptions to overcome the difficulties caused by the unboundedness of $\mathbb{Z}^{m}$ and the lack of periodic conditions. In particular, condition $\left(F_{3}\right)$ is a new superquadratic condition introduced by Tang and Wu [29].

$\left(\mathbf{V}_{\mathbf{1}}\right) \quad V=\left\{v_{n}\right\}_{n \in \mathbb{Z}^{m}}$ satisfies

$$
\lim _{|n| \rightarrow+\infty} v_{n}=+\infty
$$

(F) $f_{n} \in C(\mathbb{R}, \mathbb{R}), \lim _{|s| \rightarrow 0} \frac{f_{n}(s)}{s}=0$.

(F) $\lim _{|s| \rightarrow+\infty} \frac{F_{n}(s)}{|s|^{2}}=+\infty$ for all $n \in \mathbb{Z}^{m}$, where $F_{n}(s):=\int_{0}^{s} f_{n}(t) d t,(n, s) \in \mathbb{Z}^{m} \times \mathbb{R}$.

(F) $\widetilde{F}_{n}(s)=f_{n}(s) s-2 F_{n}(s) \geq 0$ for all $(n, s) \in \mathbb{Z}^{m} \times \mathbb{R}$, and there exist $b>0$ and $r_{\infty}>0$ such that

$$
\widetilde{F}_{n}(s) \geq b \frac{F_{n}(s)}{|s|^{2}}, \quad \forall n \in \mathbb{Z}^{m}, \forall|s| \geq r_{\infty}
$$

(F) $F_{n}(0) \equiv 0$ for all $n \in \mathbb{Z}^{m}$.

$\left(\mathbf{F}_{5}\right)$ There is $L>0$ such that $\sup _{n \in \mathbb{Z}^{m},|s|=r_{\infty}} F_{n}(s) \leq L$.

Theorem 1.1 Equation (1.1) possesses at least one nontrivial homoclinic solution $u$ if conditions $\left(V_{1}\right)$ and $\left(F_{1}\right)-\left(F_{5}\right)$ hold. Here, $u$ is nontrivial, that is, $u_{n} \not \equiv 0$.

Theorem 1.2 Equation (1.1) has infinitely many nontrivial homoclinic solutions if conditions $\left(V_{1}\right)$ and $\left(F_{1}\right)-\left(F_{5}\right)$ hold and $f_{n}(-s)=-f_{n}(s)$ for all $(n, s) \in \mathbb{Z}^{m} \times \mathbb{R}$.

Example 1.1 We give the following example to explain the rationality of the assumptions for the nonlinear terms $f_{n}$. Let

$$
F_{n}(s)= \begin{cases}a_{n}\left[c_{1}|s|^{6}+c_{2}|s|^{4}\right], & |s| \leq 2, \\ a_{n}\left[|s|^{2} \ln \left(1+|s|^{2}\right)+\sin |s|^{2}-\ln \left(1+|s|^{2}\right)\right], & |s|>2,\end{cases}
$$

where $s \in \mathbb{R}, 0<\inf _{n \in \mathbb{Z}^{m}} a_{n}<\sup _{n \in \mathbb{Z}^{m}} a_{n}<+\infty$, and $c_{1}, c_{2}>0$ are two suitable constants. It is not hard to check that it satisfies our conditions $\left(F_{1}\right)-\left(F_{5}\right)$.

Remark 1.1 (Comparisons) We give detailed comparisons between our results and the results $[18-20,23,25,26,28]$ for infinite $m$-dimensional lattices. Our Theorems 1.1 and 1.2 generalize the results mentioned. 
(1) The results of $[18,20,23,25,26,28]$ are all based on conditions $\left(V_{1}\right)$ and $\left(\mathbf{F}_{1}^{\prime}\right) f_{n} \in C(\mathbb{R}, \mathbb{R}), \lim _{|s| \rightarrow 0} \frac{f_{n}(s)}{s}=0$, and there are $a_{1}>0$ and $v>2$ such that

$$
\left|f_{n}(s)\right| \leq a_{1}\left(1+|s|^{\nu-1}\right), \quad \forall(n, s) \in \mathbb{Z}^{m} \times \mathbb{R} .
$$

However, we remove condition (1.5).

(2) The authors of $[25,26]$ used the Ambrosetti-Rabinowitz superlinear condition

$$
0<v^{\prime} F_{n}(s) \leq f_{n}(s) s \quad \text { for some } v^{\prime}>2, \forall s \in \mathbb{R} \backslash\{0\} .
$$

Obviously, (1.6) is stronger than our condition $\left(F_{2}\right)$. Besides, the authors of [20, 23, 28] used the condition

$$
\frac{f_{n}(s)}{s} \quad \text { is increasing for } s>0 \text { and decreasing for } s<0,
$$

the authors of [18] used the condition

$$
\liminf _{|s| \rightarrow+\infty} \frac{f_{n}(s) s-2 F_{n}(s)}{|s|^{\varrho}} \geq b \quad \text { for some } b>0, \varrho>\max \{1, v-2\}, \quad \forall n \in \mathbb{Z}^{m}
$$

and the authors of [19] used the condition

$$
\mu F_{n}(s) \leq f_{n}(s) s+\kappa s^{2}, \quad \mu>2, \kappa>0, \forall(n, s) \in \mathbb{Z}^{m} \times \mathbb{R} .
$$

It is not hard to check that the functions in our Example 1.1 do not satisfy conditions (1.6)-(1.9), but they all satisfy our conditions $\left(F_{1}\right)-\left(F_{5}\right)$. Therefore, our results extend those in the papers mentioned.

In Section 2, we establish the variational framework of (1.1) and give some preliminary lemmas. In Sections 3 and 4, we give detailed proofs of Theorems 1.1 and 1.2, respectively.

\section{Variational structure and preliminary lemmas}

Let

$$
\begin{aligned}
l^{p} & \equiv l^{p}\left(\mathbb{Z}^{m}\right) \\
& :=\left\{u=\left\{u_{n}\right\}: n \in \mathbb{Z}^{m}, u_{n} \in \mathbb{R},\|u\|_{l^{p}}=\left(\sum_{n \in \mathbb{Z}^{m}}\left|u_{n}\right|^{p}\right)^{1 / p}<\infty\right\}, \quad p \in[1,+\infty),
\end{aligned}
$$

be real sequence spaces. The following elementary embedding relations hold:

$$
l^{p} \subset l^{q}, \quad\|u\|_{l^{q}} \leq\|u\|_{l^{p}}, \quad 1 \leq p \leq q \leq \infty, \quad \text { where }\|u\|_{l^{\infty}}:=\max _{n \in \mathbb{Z}^{m}}\left|u_{n}\right| .
$$

Let $L:=-\triangle+V$ be defined by $L u_{n}:=-\triangle u_{n}+v_{n} u_{n}$ for $u \in l^{2}$. Let $E$ be the form domain of $L$, that is, $E:=\mathcal{D}\left(L^{1 / 2}\right)$ (the domain of $L^{1 / 2}$ ). Under our assumptions, the operator $L$ is an unbounded self-adjoint operator in $l^{2}$. Since the operator $-\triangle$ is bounded in $l^{2}$, it is easy to 
see that $E=\left\{u \in l^{2}: V^{1 / 2} u \in l^{2}\right\}$, where $V^{1 / 2} u$ is defined by $V^{1 / 2} u_{n}:=v_{n}^{1 / 2} u_{n}$ for $u \in l^{2}$. We define respectively on $E$ the inner product and norm by

$$
(u, v)_{E}:=(u, v)_{l^{2}}+\left(L^{1 / 2} u, L^{1 / 2} v\right)_{l^{2}} \quad \text { and } \quad\|u\|_{E}=(u, u)_{E}^{1 / 2}
$$

where $(u, v)_{l^{2}}$ is the inner product in $l^{2}$. Then $E$ is a Hilbert space.

Lemma 2.1 ([26]) If (1.3) holds, then we have:

(1) The embedding maps from $E$ into $l^{p}$ are compact for all $p \in[2, \infty]$, and there exist $\gamma_{q}>0$ such that

$$
\|u\|_{l^{q}} \leq \gamma_{q}\|u\|, \quad \forall u \in E .
$$

(2) The spectrum $\sigma(L-\omega)$ consists of the eigenvalues:

$$
\lambda_{1}-\omega<\lambda_{2}-\omega<\cdots<\lambda_{k}-\omega<\cdots \rightarrow+\infty .
$$

Let $e_{k}$ be the eigenfunctions with $(L-\omega) e_{k}=\left(\lambda_{k}-\omega\right) e_{k}$ and $\left\|e_{k}\right\|_{l^{2}}=1, k=1,2, \ldots$ Moreover, $\left\{e_{k}: k=1,2, \ldots\right\}$ is an orthonormal basis of $l^{2}$. Let $\sharp(D)$ denote the number of $i$ such that $i \in D$. Let

$$
\begin{aligned}
& k_{1}:=\sharp\left(\left\{i: \lambda_{i}-\omega<0\right\}\right), \\
& k_{0}:=\sharp\left(\left\{i: \lambda_{i}-\omega=0\right\}\right), \\
& k_{2}:=k_{0}+k_{1}
\end{aligned}
$$

and

$$
\begin{aligned}
& E^{-}:=\operatorname{span}\left\{e_{1}, \ldots, e_{k_{1}}\right\}, \\
& E^{0}:=\operatorname{span}\left\{e_{k_{1}+1}, \ldots, e_{k_{2}}\right\}, \\
& E^{+}:=\overline{\operatorname{span}\left\{e_{k_{2}+1}, \ldots\right\}},
\end{aligned}
$$

where the closure is taken with respect to the norm $\|\cdot\|_{E}$. Then we have the orthogonal decomposition

$$
E=E^{-} \oplus E^{0} \oplus E^{+}
$$

with respect to the inner product $(\cdot, \cdot)_{E}$. Now, we introduce respectively on $E$ the following inner product and norm:

$$
(u, v):=\left(u^{0}, v^{0}\right)_{l^{2}}+\left(L^{\frac{1}{2}} u, L^{\frac{1}{2}} v\right)_{l^{2}}, \quad\|u\|=(u, u)^{\frac{1}{2}}
$$

where $u, v \in E=E^{-} \oplus E^{0} \oplus E^{+}$with $u=u^{-}+u^{0}+u^{+}$and $v=v^{-}+v^{0}+v^{+}$. Clearly, the norms $\|\cdot\|$ and $\|\cdot\|_{E}$ are equivalent, and the decomposition $E=E^{-} \oplus E^{0} \oplus E^{+}$is also orthogonal with respect to both inner products $(\cdot, \cdot)$ and $(\cdot, \cdot)_{l^{2}}$. 
In view of the above arguments, we consider the functional $\Phi$ on $E$ defined by

$$
\begin{aligned}
\Phi(u) & =\frac{1}{2}((L-\omega) u, u)_{l^{2}}-\sum_{n \in \mathbb{Z}^{m}} F_{n}\left(u_{n}\right) \\
& =\frac{1}{2}\left\|u^{+}\right\|^{2}-\frac{1}{2}\left\|u^{-}\right\|^{2}-\sum_{n \in \mathbb{Z}^{m}} F_{n}\left(u_{n}\right) .
\end{aligned}
$$

Under our assumptions, $\Phi \in C^{1}(E, \mathbb{R})$, and the derivative is given by

$$
\left\langle\Phi^{\prime}(u), v\right\rangle=\left(u^{+}, v^{+}\right)-\left(u^{-}, v^{-}\right)-\sum_{n \in \mathbb{Z}^{m}} f_{n}\left(u_{n}\right) v_{n}
$$

where $u, v \in E=E^{-} \oplus E^{0} \oplus E^{+}$with $u=u^{-}+u^{0}+u^{+}$and $v=v^{-}+v^{0}+v^{+}$. The standard argument shows that nonzero critical points of $\Phi$ are nontrivial solutions of (1.1).

Definition 2.1 We say that

(1) $I \in C^{1}(X, \mathbb{R})$ satisfies $(C)$-condition if any sequence $\left\{u^{k}\right\}$ such that $I\left(u^{k}\right)$ is bounded and

$$
\left\|I^{\prime}\left(u^{k}\right)\right\|\left(1+\left\|u^{k}\right\|\right) \rightarrow 0, \quad k \rightarrow \infty
$$

has a convergent subsequence.

(2) $I \in C^{1}(X, \mathbb{R})$ satisfies $(P S)$-condition if any sequence $\left\{u^{k}\right\}$ such that $I\left(u^{k}\right)$ is bounded and

$$
I^{\prime}\left(u^{k}\right) \rightarrow 0, \quad k \rightarrow \infty
$$

has a convergent subsequence.

We shall use the following two lemmas to prove our main results:

Lemma 2.2 ([30]) Let E be a real Banach space, and let $I \in C^{1}(E, \mathbb{R})$ satisfy $(P S)$-condition. Suppose $I(0)=0$ and

(1) there are constants $\rho, \alpha>0$ such that $\left.I\right|_{\partial B_{\rho}} \geq \alpha$;

(2) there is $e \in E \backslash B_{\rho}$ such that $I(e) \leq 0$. Then I possesses a critical value $c \geq \alpha$.

Moreover, $c$ can be characterized as $c=\inf _{g \in \Gamma} \max _{u \in g([0,1])} I(u)$, where $\Gamma=\{g \in C([0,1], E) \mid g(0)=0, g(1)=e\}$.

Lemma 2.3 ([31]) Let $X$ be an infinite-dimensional Banach space such that $X=Y \oplus Z$, where $Y$ is finite-dimensional. Let $I \in C^{1}(X, \mathbb{R})$ be an invariant functional. Suppose that, for any $k \in N$, there exist $\rho_{k}>r_{k}>0$ such that

(1) I satisfies $(C)$-condition for all $c>0$;

(2) $a_{k}:=\max _{u \in Y_{k},\|u\|=\rho_{k}} I(u) \leq 0$;

(3) $b_{k}:=\inf _{u \in Y_{k},\|u\|=\rho_{k}} I(u) \rightarrow \infty, k \rightarrow \infty$.

Then I has an unbounded sequence of critical values.

Let $\left\{e_{j}\right\}_{j=1}^{\infty}$ be an orthonormal basis of $E$, and let $X_{j}:=\mathbb{R} e_{j}$. Then $Y_{k}=\bigoplus_{j=1}^{k} X_{j}=$ $\operatorname{span}\left\{e_{1}, \ldots, e_{k}\right\}$ and $Z_{k}=\overline{\bigoplus_{j=k}^{\infty} X_{j}}=\overline{\operatorname{span}\left\{e_{k}, \ldots\right\}}$ for all $k \in \mathbb{N}$. 
Lemma 2.4 If assumptions $\left(V_{1}\right)$ and $\left(F_{1}\right)-\left(F_{5}\right)$ hold, then $\Phi$ satisfies $(C)$-condition.

Proof We assume that, for any sequence $\left\{u^{k}\right\} \subset E$, $\Phi\left(u^{k}\right)$ is bounded and $\left\|\Phi^{\prime}\left(u^{k}\right)\right\|(1+$ $\left.\left\|u^{k}\right\|\right) \rightarrow 0$. Then there exists a constant $M>0$ such that

$$
\left|\Phi\left(u^{k}\right)\right| \leq M, \quad\left\|\Phi^{\prime}\left(u^{k}\right)\right\|\left(1+\left\|u^{k}\right\|\right) \leq M .
$$

(i) First, we prove the boundedness of $\left\{u^{k}\right\}$. If not, then $\left\|u^{k}\right\| \rightarrow \infty$ as $k \rightarrow \infty$. Let $v^{k}=$ $\frac{u^{k}}{\left\|u^{k}\right\|}$. Then $\left\|v^{k}\right\|=1$. We can assume that $v^{k} \rightarrow v=\left\{v_{n}\right\}_{n \in \mathbb{Z}^{m}}$ in $E$ passing to a subsequence, which, together with Lemma 2.1, implies $v^{k} \rightarrow v$ in $l^{q}$ for $2 \leq q<\infty$ and $v_{n}^{k} \rightarrow v_{n}$ for all $n \in \mathbb{Z}^{m}$. By the space decomposition we have

$$
\|u\|^{2}=\left\|u^{+}\right\|^{2}+\left\|u^{-}+u^{0}\right\|^{2}
$$

Then, by (2.2), (2.5), and (2.6) we have

$$
\sum_{n \in \mathbb{Z}^{m}} \frac{F_{n}\left(u_{n}^{k}\right)}{\left\|u^{k}\right\|^{2}}=\frac{1}{2}-\frac{\Phi\left(u^{k}\right)}{\left\|u^{k}\right\|^{2}}-\frac{1}{2} \frac{\left\|\left(u^{k}\right)^{-}\right\|^{2}+\left\|\left(u^{k}\right)^{-}+\left(u^{k}\right)^{0}\right\|^{2}}{\left\|u^{k}\right\|^{2}},
$$

which implies that, for $k$ large enough, we have

$$
\sum_{n \in \mathbb{Z}^{m}} \frac{F_{n}\left(u_{n}^{k}\right)}{\left\|u^{k}\right\|^{2}} \leq \frac{1}{2}+\frac{M}{\left\|u^{k}\right\|^{2}} \leq 1 .
$$

If $v \neq 0$, then we let $A:=\left\{n \in \mathbb{Z}^{m}:\left|v_{n}\right|>0\right\}$. For all $n \in A$, by $v_{n}^{k}=\frac{u_{n}^{k}}{\left\|u^{k}\right\|}$ and $\left\|u^{k}\right\| \rightarrow \infty$ we have $\lim _{k \rightarrow \infty}\left|u_{n}^{k}\right|=\infty$. It follows from $\left(F_{2}\right)$ that $F_{n}(s) \geq 0$ for all $(n, s) \in \mathbb{Z}^{m} \times \mathbb{R}$ (see AX 1 in Appendix) and the Fatou lemma that

$$
\begin{aligned}
\lim _{k \rightarrow \infty} \sum_{n \in \mathbb{Z}^{m}} \frac{F_{n}\left(u_{n}^{k}\right)}{\left\|u^{k}\right\|^{2}} & \geq \lim _{k \rightarrow \infty} \sum_{n \in A} \frac{F_{n}\left(u_{n}^{k}\right)}{\left\|u^{k}\right\|^{2}} \\
& =\lim _{k \rightarrow \infty} \sum_{n \in A} \frac{F_{n}\left(u_{n}^{k}\right)}{\left|u_{n}^{k}\right|^{2}}\left|v_{n}^{k}\right|^{2} \\
& =+\infty,
\end{aligned}
$$

which contradicts with (2.8). So, in this case, $\left\{u^{k}\right\}$ is bounded in $E$.

If $v=0$, then $v^{k} \rightarrow 0$ in $l^{q}, 2 \leq q<\infty$, and $v_{n}^{k} \rightarrow 0$ for all $n \in \mathbb{Z}^{m}$. Since $\operatorname{dim}\left(E^{-} \oplus E^{0}\right)<\infty$, it follows from (2.5) and (2.7) that, for $k$ large enough, there exists a constant $l_{0}>0$ such that

$$
\begin{aligned}
\sum_{n \in \mathbb{Z}^{m}} \frac{F_{n}\left(u_{n}^{k}\right)}{\left\|u^{k}\right\|^{2}} & \geq \frac{1}{2}-\frac{M}{\left\|u^{k}\right\|^{2}}-\frac{l_{0}}{2} \sum_{n \in \mathbb{Z}^{m}}\left(\frac{\left|\left(u_{n}^{k}\right)^{-}\right|^{2}+\left|\left(u_{n}^{k}\right)^{-}+\left(u_{n}^{k}\right)^{0}\right|^{2}}{\left\|u^{k}\right\|^{2}}\right) \\
& =\frac{1}{2}-\frac{M}{\left\|u^{k}\right\|^{2}}-\frac{l_{0}}{2} \sum_{n \in \mathbb{Z}^{m}}\left(\left|\left(v_{n}^{k}\right)^{-}\right|^{2}+\left|\left(v_{n}^{k}\right)^{-}+\left(v_{n}^{k}\right)^{0}\right|^{2}\right) \\
& \geq \frac{1}{4} .
\end{aligned}
$$


Then by $\left(F_{1}\right)$, for any $\varepsilon>0$, there exists $\sigma>0$ such that

$$
\left|f_{n}(s)\right| \leq \varepsilon|s|, \quad|s| \leq \sigma, n \in \mathbb{Z}^{m}
$$

It follows from $F_{n}(s) \geq 0$ for all $(n, s) \in \mathbb{Z}^{m} \times \mathbb{R}$ (see AX 1 in Appendix) and $\left(F_{4}\right)$ that, for all $n \in \mathbb{Z}^{m}$ and $|s| \leq \sigma$,

$$
\begin{aligned}
F_{n}(s) & =\left|F_{n}(s)-F_{n}(0)\right| \\
& =\left|\int_{0}^{1} f_{n}(t s) s d t\right| \\
& \leq \int_{0}^{1}\left|f_{n}(t s)\right||s| d t \\
& \leq \int_{0}^{1} \varepsilon|t s||s| d t \leq \varepsilon|s|^{2} .
\end{aligned}
$$

Let $\varepsilon=1$. Then there exists $\sigma_{0}>0$ such that (2.10) holds for all $n \in \mathbb{Z}^{m}$ and $|s| \leq \sigma_{0}$. By $\left(F_{3}\right)$ we have $\frac{d}{d \vartheta} \vartheta^{-2} F_{n}(\vartheta s) \geq 0$ for all $\vartheta \geq 1$, so $\vartheta^{-2} F_{n}(\vartheta s)$ is nondecreasing in $\vartheta$ for $\vartheta \geq 1$. Then by $\left(F_{5}\right)$, for all $|s| \leq r_{\infty}$, we have

$$
F_{n}(s) \leq F_{n}\left(\frac{r_{\infty} s}{|s|}\right)\left(\frac{|s|}{r_{\infty}}\right)^{2} \leq F_{n}\left(\frac{r_{\infty} s}{|s|}\right) \leq L .
$$

Then since $F_{n}(s) \geq 0$ for all $(n, s) \in \mathbb{Z}^{m} \times \mathbb{R}$ (see $\mathrm{AX} 1$ in Appendix), by $\left(F_{3}\right),(2.3)$, (2.5), (2.10), (2.11), and the Sobolev embedding theorem, for $k$ large enough, we have

$$
\begin{aligned}
0 \leq & \sum_{n \in \mathbb{Z}^{m}} \frac{F_{n}\left(u_{n}^{k}\right)}{\left\|u^{k}\right\|^{2}} \\
\leq & \sum_{\left\{n \in \mathbb{Z}^{m},\left|u^{k}\right|>r_{\infty}\right\}} \frac{F_{n}\left(u_{n}^{k}\right)}{\left|u_{n}^{k}\right|^{2}}\left|v_{n}^{k}\right|^{2}+\sum_{\left\{n \in \mathbb{Z}^{m},\left|u^{k}\right| \leq \sigma_{0}\right\}} \frac{F_{n}\left(u_{n}^{k}\right)}{\left\|u^{k}\right\|^{2}} \\
& +\sum_{\left\{n \in \mathbb{Z}^{m}, \sigma_{0} \leq\left|u^{k}\right| \leq r_{\infty}\right\}} \frac{F_{n}\left(u_{n}^{k}\right)}{\left\|u^{k}\right\|^{2}} \\
\leq & \left\|v^{k}\right\|_{l^{\infty}}^{2} \sum_{\left\{n \in \mathbb{Z}^{m},\left|u^{k}\right|>r_{\infty}\right\}} \frac{F_{n}\left(u_{n}^{k}\right)}{\left|u_{n}^{k}\right|^{2}}+\sum_{\left\{n \in \mathbb{Z}^{m},\left|u^{k}\right| \leq \sigma_{0}\right\}}\left|v_{n}^{k}\right|^{2} \\
& +\sum_{\left\{n \in \mathbb{Z}^{m}, \sigma_{0} \leq\left|u^{k}\right| \leq r_{\infty}\right\}} \frac{F_{n}\left(u_{n}^{k}\right)\left|u_{n}^{k}\right|^{2}}{\sigma_{0}^{2}\left\|u^{k}\right\|^{2}} \\
\leq & \frac{\left\|v^{k}\right\|_{l^{\infty}}^{2}}{b} \sum_{\left\{n \in \mathbb{Z}^{m},\left|u^{k}\right|>r_{\infty}\right\}} \widetilde{F}_{n}\left(u_{n}^{k}+\left(1+\frac{L}{\sigma_{0}^{2}}\right)\left\|v^{k}\right\|_{l^{2}}^{2}\right. \\
\leq & \frac{\left\|v^{k}\right\|_{l^{\infty}}^{2}}{b}\left(2 \Phi\left(u^{k}\right)-\left\langle\Phi^{\prime}\left(u^{k}\right), u^{k}\right\rangle\right)+\left(1+\frac{L}{\sigma_{0}^{2}}\right)\left\|v^{k}\right\|_{l^{2}}^{2} \\
\leq & \frac{3 M}{b}\left\|v^{k}\right\|_{l^{\infty}}^{2}+\left(1+\frac{L}{\sigma_{0}^{2}}\right)\left\|v^{k}\right\|_{l^{2}}^{2} \rightarrow 0 .
\end{aligned}
$$

Clearly, (2.12) contradicts with (2.9). Thus $\left\|u^{k}\right\|$ is still bounded in this case. 
(ii) Second, we prove that $\left\{u^{k}\right\}$ has a convergent subsequence in $E$. The boundedness of $\left\{u^{k}\right\}$ implies that $u^{k} \rightarrow u$ in $E^{+}$passing to a subsequence, where $u=\left\{u_{n}\right\}_{n \in \mathbb{Z}^{m}}$. Now we have

$$
\sum_{n \in \mathbb{Z}^{m}}\left[f_{n}\left(u_{n}^{k}\right)\left(u_{n}^{k}-u_{n}\right)\right] \rightarrow 0, \quad k \rightarrow \infty
$$

Note that Lemma 2.1 implies that $u^{k} \rightarrow u$ in $l^{q}$ for all $2 \leq q<\infty$, so we have

$$
\left\|u^{k}-u\right\|_{l^{2}} \rightarrow 0
$$

The boundedness of $\left\{u^{k}\right\}$ and Lemma 2.1 imply that $\left\|u^{k}\right\|_{q}<\infty$ for all $2 \leq q<\infty$. Then by $\left(F_{1}\right),(2.14)$, and the Hölder inequality, for $\varepsilon>0$, there exists $\delta>0$ such that, for $|s|<\delta$, we have

$$
\begin{aligned}
\left|\sum_{n \in \mathbb{Z}^{m}}\left[f_{n}\left(u_{n}^{k}\right)\left(u_{n}^{k}-u_{n}\right)\right]\right| & \leq \sum_{n \in \mathbb{Z}^{m}}\left|f_{n}\left(u_{n}^{k}\right)\left(u_{n}^{k}-u_{n}\right)\right| \\
& \leq \sum_{n \in \mathbb{Z}^{m}}\left(\varepsilon\left|u_{n}^{k}\right|\left|u_{n}^{k}-u_{n}\right|\right) \\
& =\varepsilon \sum_{n \in \mathbb{Z}^{m}}\left(\left|u_{n}^{k}\right|\left|u_{n}^{k}-u_{n}\right|\right) \\
& \leq \varepsilon\left\|u^{k}\right\|_{l^{2}}\left\|u^{k}-u\right\|_{l^{2}} \rightarrow 0 .
\end{aligned}
$$

So (2.13) holds. Therefore, since $\Phi^{\prime}\left(u^{k}\right) \rightarrow 0, u^{k} \rightarrow u$ in $E^{+}$, by (2.13) and the definition of $\Phi^{\prime}$ we have

$$
\begin{aligned}
0 & =\lim _{k \rightarrow \infty}\left\langle\Phi^{\prime}\left(u^{k}\right), u^{k}-u\right\rangle \\
& =\lim _{k \rightarrow \infty}\left(u^{k}, u^{k}-u\right)-\lim _{k \rightarrow \infty} \sum_{n \in \mathbb{Z}^{m}}\left(f_{n}\left(u_{n}^{k}\right)\left(u_{n}^{k}-u_{n}\right)\right) \\
& =\lim _{k \rightarrow \infty}\left\|u^{k}\right\|^{2}-\|u\|^{2}-0
\end{aligned}
$$

that is,

$$
\lim _{k \rightarrow \infty}\left\|u^{k}\right\|=\|u\|
$$

Since $u^{k} \rightarrow u$ in $E^{+}$, it follows that

$$
\left\|u^{k}-u\right\|^{2}=\left(u^{k}-u, u^{k}-u\right) \rightarrow 0
$$

that is, $\left\{u^{k}\right\}$ has a convergent subsequence in $E^{+}$. Since $\operatorname{dim}\left(E^{-} \oplus E^{0}\right)<\infty$, it follows that $\left\{u^{k}\right\}$ has a convergent subsequence in $E$. Thus $\Phi$ satisfies $(C)$-condition.

\section{Proof of Theorem 1.1}

Lemma 3.1 If assumptions $\left(V_{1}\right),\left(F_{1}\right),\left(F_{3}\right)$, and $\left(F_{4}\right)$ hold, then there exist constants $\varrho, \alpha>0$ such that $\left.\Phi\right|_{S} \geq \alpha$, where $S=\left\{u \in E^{+} \mid\|u\|=\varrho\right\}$. 
Proof In view of (2.9), let $\varepsilon=\frac{1}{4 \gamma_{2}^{2}}$, where $\gamma_{2}$ is defined in Lemma 2.1. Then there exists $\sigma_{1}>0$ such that $F_{n}(s) \leq \varepsilon|s|^{2}$ for all $n \in \mathbb{Z}^{m}$ and $|s| \leq \sigma_{1}$. Let

$$
\rho=\frac{\sigma_{1}}{\gamma_{\infty}}, \quad \alpha=\frac{1}{4} \varrho^{2}>0,
$$

where $\gamma_{\infty}$ is defined in Lemma 2.1. This implies that $0<\|u\|_{l} \leq \sigma_{1}$ for all $u \in S$. Then by (2.2) we have

$$
\begin{aligned}
\Phi(u) & =\frac{1}{2}\|u\|^{2}-\sum_{n \in \mathbb{Z}^{m}} F_{n}\left(u_{n}\right) \\
& \geq \frac{1}{2}\|u\|^{2}-\frac{1}{4 \gamma_{2}^{2}} \sum_{n \in \mathbb{Z}^{m}}\left|u_{n}\right|^{2} \\
& \geq \frac{1}{2}\|u\|^{2}-\frac{1}{4}\|u\|^{2}=\frac{1}{4}\|u\|^{2} .
\end{aligned}
$$

Thus, by (3.1) and the definitions of $\varrho$ and $\alpha$, the proof of the lemma is finished.

Lemma 3.2 If assumptions $\left(V_{1}\right)$ and $\left(F_{1}\right)-\left(F_{3}\right)$ hold, then there exists $\zeta>\varrho$ such that $\left.\Phi\right|_{\partial Q} \leq$ 0 with $\varrho$ defined in Lemma 3.1.

Proof Let $e \in E^{+}$with $\|e\|=1$ and $K=E^{-} \oplus E^{0} \oplus \operatorname{span}\{e\}$. Then there exists a small enough constant $\varepsilon_{1}>0$ such that

$$
\sharp\left(\left\{n \in \mathbb{Z}^{m}:\left|u_{n}\right| \geq \varepsilon_{1}\|u\|\right\}\right) \geq 1, \quad \forall u \in K \backslash\{0\} .
$$

The detailed proof of (3.2) is given in the Appendix.

For $u=u^{+}+u^{0}+u^{-} \in K$, let $\Omega_{u}=\left\{n \in \mathbb{Z}^{m}|| u_{n} \mid \geq \varepsilon_{1}\|u\|\right\}$. By $\left(F_{2}\right)$, for $M=2 / \varepsilon_{1}^{2}>0$, there exists $L_{1}>0$ such that

$$
F_{n}(s) \geq M|s|^{2}, \quad \forall|s| \geq L_{1}, \forall n \in \mathbb{Z}^{m} .
$$

Thus we have

$$
F_{n}\left(u_{n}\right) \geq M\left|u_{n}\right|^{2} \geq M \varepsilon_{1}^{2}\|u\|^{2}, \quad \forall n \in \Omega_{u},
$$

where $u \in K$ and $\|u\| \geq L_{1} / \varepsilon_{1}$. It follows from $\left(F_{3}\right)$ and (3.2) that

$$
\begin{aligned}
\Phi(u) & =\frac{1}{2}\left\|u^{+}\right\|^{2}-\frac{1}{2}\left\|u^{-}\right\|^{2}-\sum_{n \in \mathbb{Z}^{m}} F_{n}\left(u_{n}\right) \\
& \leq \frac{1}{2}\left\|u^{+}\right\|^{2}-\sum_{n \in \Omega_{u}} F_{n}\left(u_{n}\right) \\
& \leq \frac{1}{2}\left\|u^{+}\right\|^{2}-M \varepsilon_{1}^{2}\|u\|^{2} \cdot \sharp\left(\Omega_{u}\right) \\
& \leq \frac{1}{2}\left\|u^{+}\right\|^{2}-M \varepsilon_{1}^{2}\|u\|^{2} \leq 0
\end{aligned}
$$


for all $u \in K$ and $\|u\| \geq L_{1} / \varepsilon_{1}$. Let $Q=\{\eta e \mid 0 \leq \eta \leq \zeta\} \oplus\left\{u \in E^{-} \oplus E^{0} \mid\|u\| \leq \zeta\right\}$. Then we have

$$
\partial Q=Q_{1} \cup Q_{2} \cup Q_{3},
$$

where

$$
\begin{aligned}
& Q_{1}=\left\{u \in E^{-} \oplus E^{0} \mid\|u\| \leq \zeta\right\}, \\
& Q_{2}=\zeta e \oplus\left\{u \in E^{-} \oplus E^{0} \mid\|u\| \leq \zeta\right\}, \\
& Q_{3}=\{\eta e \mid 0 \leq \eta \leq \zeta\} \oplus\left\{u \in E^{-} \oplus E^{0} \mid\|u\|=\zeta\right\} .
\end{aligned}
$$

Then by (3.3), for all $\zeta \geq L_{1} / \varepsilon_{1}$, we have $\Phi(u) \leq 0$ for all $u \in Q_{2} \cup Q_{3}$. It follows from $\left(F_{3}\right)$ that $\Phi(u) \leq 0$ for all $u \in E^{-} \oplus E^{0}$, which implies that $\Phi(u) \leq 0$ for all $u \in Q_{1}$. Thus, for all $\zeta>\max \left\{\varrho, L_{1} / \varepsilon_{1}\right\}$, we have

$$
\Phi(u) \leq 0, \quad \forall u \in \partial Q
$$

The proof is finished.

Proof of Theorem 1.1 Similarly to Lemma 2.4, we can also prove that $\Phi$ satisfies (PS)condition. Then by Lemmas 3.1 and 3.2 conditions (1) and (2) of Lemma 2.2 hold, so Lemma 2.2 implies that $\Phi$ possesses a critical point $u$ such that $\Phi(u) \geq \alpha$. Therefore $u$ is a homoclinic solution of problem (1.1).

\section{Proof of Theorem 1.2}

Let

$$
\beta_{k}(p)=\sup _{u \in Z_{k},\|u\|=1}\|u\|_{l^{p}}, \quad k \in N, q \in[2,+\infty]
$$

Lemma 4.1 If assumptions $\left(V_{1}\right),\left(F_{1}\right)$, and $\left(F_{3}\right)-\left(F_{5}\right)$ hold, then $b_{k} \rightarrow \infty$ as $k \rightarrow \infty$, where

$$
b_{k}=\inf _{u \in Z_{k},\|u\|=\tau_{k}} \Phi(u), \quad \tau_{k}=r_{\infty} / \beta_{k}(\infty) .
$$

Proof By Lemma 2.1, $E$ is embedded compactly into $l^{p}$. Then $\beta_{k}(p) \rightarrow 0$ as $k \rightarrow \infty$. For $k$ large enough, we choose $k$ such that $Z_{k} \subset E^{+}$. Note that $\tau_{k} \rightarrow+\infty$ as $k \rightarrow \infty$, and so for any $u \in Z_{k}$ with $\|u\|=\tau_{k}$, we have

$$
\|u\|_{l^{\infty}} \leq r_{\infty}
$$

Then by (2.10), there exists $r_{\infty} \geq \sigma_{2}>0$, for all $\left|u_{n}\right| \leq \sigma_{2}$, we have

$$
F_{n}\left(u_{n}\right) \leq\left|u_{n}\right|^{2}
$$


Then by (2.2), (2.11), (4.1), and (4.2), for any $u \in Z_{k}$ with $\|u\|=\tau_{k}$, we have

$$
\begin{aligned}
\Phi(u) & =\frac{1}{2}\|u\|^{2}-\sum_{n \in \mathbb{Z}^{m}} F_{n}\left(u_{n}\right) \\
& =\frac{1}{2}\|u\|^{2}-\sum_{n \in \mathbb{Z}^{m},\left|u_{n}\right| \leq \sigma_{2}} F_{n}\left(u_{n}\right)-\sum_{n \in \mathbb{Z}^{m}, \sigma_{2} \leq\left|u_{n}\right| \leq r_{\infty}} F_{n}\left(u_{n}\right) \\
& \geq \frac{1}{2}\|u\|^{2}-\sum_{n \in \mathbb{Z}^{m},\left|u_{n}\right| \leq \sigma_{2}}\left|u_{n}\right|^{2}-\sum_{n \in \mathbb{Z}^{m}, \sigma_{2} \leq\left|u_{n}\right| \leq r_{\infty}} \frac{\left.u_{n}\right|^{2}}{\sigma_{2}^{2}} F_{n}\left(u_{n}\right) \\
& \geq \frac{1}{2}\|u\|^{2}-\|u\|_{l^{2}}^{2}-L \sum_{n \in \mathbb{Z}^{m}, \sigma_{2} \leq\left|u_{n}\right| \leq r_{\infty}} \frac{\left|u_{n}\right|^{2}}{\sigma_{2}^{2}} \\
& =\frac{1}{2}\|u\|^{2}-\|u\|_{l^{2}}^{2}-\frac{L}{\sigma_{2}^{2}} \sum_{n \in \mathbb{Z}^{m}, \sigma_{2} \leq\left|u_{n}\right| \leq r_{\infty}}\left|u_{n}\right|^{2} \\
& =\frac{1}{2}\|u\|^{2}-\left(1+\frac{L}{\sigma_{2}^{2}}\right)\|u\|^{2} \\
& \geq \frac{1}{2}\|u\|^{2}-\left(1+\frac{L}{\sigma_{2}^{2}}\right)\left(\beta_{k}(2)\right)^{2}\|u\|_{l^{2}}^{2} \\
\geq & \frac{1}{4}\|u\|^{2}=\frac{1}{4} \tau_{k}^{2}, \quad k>k_{0}, k_{0} \in N .
\end{aligned}
$$

Then we have

$$
b_{k}=\inf _{u \in Z_{k},\|u\|=\tau_{k}} \Phi(u) \geq \frac{1}{4} \tau_{k}^{2} \rightarrow+\infty, \quad k \rightarrow \infty .
$$

So the lemma is proved.

Lemma 4.2 If assumptions $\left(V_{1}\right)$ and $\left(F_{2}\right)$ hold, then we have $a_{k} \leq 0$ for all $k \in N$, where

$$
a_{k}=\max _{u \in Y_{k},\|u\|=\rho_{k}} \Phi(u)
$$

Proof For any $u \in Y_{k} /\{0\}, \delta>0$, where $u=u^{-}+u^{0}+u^{+}$, let

$$
\Gamma_{\delta}(u)=\left\{n \in \mathbb{Z}^{m}:\left|u_{n}\right| \geq \delta\|u\|\right\}
$$

By (3.2), for all $u \in Y_{k} /\{0\}$, we obtain that there exists $\varepsilon_{1}>0$ such that

$$
\sharp\left(\Gamma_{\varepsilon_{1}}(u)\right) \geq 1
$$

By $\left(F_{2}\right)$, there exists $\gamma_{2}>0$ such that, for all $u \in Y_{k}$ and $n \in \Gamma_{\varepsilon_{1}}(u)$ with $\|u\| \geq \gamma_{2}$, we have

$$
F_{n}\left(u_{n}\right) \geq \frac{1}{\varepsilon_{1}^{2}}\left|u_{n}\right|^{2} \geq\|u\|^{2}
$$


We choose $\rho_{k}>\max \left\{\gamma_{2}, \tau_{k}\right\}$. It follows from (2.2), (4.3), (4.4), and $F_{n}(s) \geq 0$ for all $(n, s) \in$ $\mathbb{Z}^{m} \times \mathbb{R}$ (see AX 1 in Appendix) that, for any $u \in Y_{k}$ with $\|u\|=\rho_{k}$,

$$
\begin{aligned}
\Phi(u) & =\frac{1}{2}\left\|u^{+}\right\|^{2}-\left(\frac{1}{2}\left\|u^{-}\right\|^{2}+\sum_{n \in \mathbb{Z}^{m}} F_{n}\left(u_{n}\right)\right) \\
& \leq \frac{1}{2}\|u\|^{2}-\sum_{n \in \Gamma_{\varepsilon_{1}}(u)} F_{n}\left(u_{n}\right) \\
& \leq \frac{1}{2}\|u\|^{2}-\|u\|^{2} \sharp\left(\Gamma_{\varepsilon_{1}}(u)\right) \\
& \leq-\frac{1}{2}\|u\|^{2} \leq 0,
\end{aligned}
$$

which means that $a_{k} \leq 0$ for all $k \in N$, and the proof of the lemma is finished.

Proof of Theorem 1.2 Let $X=E, Y=Y_{m}$, and $Z=Z_{m}$. By Lemma 2.4, under our assumptions, $\Phi$ satisfies $(C)$-condition. Clearly, condition (1) of Lemma 2.3 holds. Besides, conditions (2) and (3) of Lemma 2.3 hold by Lemmas 4.1 and 4.2, respectively. So problem (1.1) possesses infinitely many nontrivial solutions by Lemma 2.3. Therefore, Theorem 1.2 is true.

\section{Appendix}

In this section, we prove the following facts.

AX 1 If assumptions $\left(F_{1}\right),\left(F_{3}\right)$, and $\left(F_{4}\right)$ hold, then $F_{n}(s) \geq 0$ for all $(n, s) \in \mathbb{Z}^{m} \times \mathbb{R}$.

Proof

$$
g(t)=\frac{F_{n}(t s)}{t^{2}}, \quad(n, s) \in \mathbb{Z}^{m} \times \mathbb{R}, t>0 .
$$

By condition $\left(F_{3}\right)$ we have

$$
g^{\prime}(t)=\frac{f_{n}(t s) t s-2 F_{n}(t s)}{t^{3}} \geq 0, \quad(n, s) \in \mathbb{Z}^{m} \times \mathbb{R},
$$

which implies that $g(t)$ is nondecreasing in $(0,+\infty)$. By $\left(F_{1}\right)$ and $\left(F_{4}\right)$ we have

$$
\lim _{t \rightarrow 0}|g(t)|=\lim _{t \rightarrow 0}\left|\frac{F_{n}(t s)}{t^{2}}\right|=\lim _{t \rightarrow 0}\left|\frac{f_{n}(t s) s}{2 t}\right| \leq \lim _{t \rightarrow 0} \frac{\left|f_{n}(t s)\right|}{t s} \frac{|s|^{2}}{2}=0
$$

for all $(n, s) \in \mathbb{Z}^{m} \times \mathbb{R} /\{0\}$. Thus $\lim _{t \rightarrow 0} g(t)=0$, which shows that $g(t) \geq 0$ for all $t>0$ and $(n, s) \in \mathbb{Z}^{m} \times \mathbb{R} /\{0\}$. It follows from $\left(F_{4}\right)$ that

$$
F_{n}(s)=g(1) \geq 0, \quad(n, s) \in \mathbb{Z}^{m} \times \mathbb{R} .
$$

Thus the proof is finished.

AX 2 The fact (3.2) in Lemma 3.2 holds. 
Proof If not, for any positive integer $k$, there exists $u^{k} \in K \backslash\{0\}$ such that

$$
\sharp\left(\left\{n \in \mathbb{Z}^{m}:\left|u_{n}^{k}\right| \geq \frac{1}{k}\left\|u^{k}\right\|\right\}\right)=0 .
$$

Let $v^{k}=u^{k} /\left\|u^{k}\right\|$, then $\left\|v^{k}\right\|=1$, and for all $k$, we have

$$
\sharp\left(\left\{n \in \mathbb{Z}^{m}:\left|v_{n}^{k}\right| \geq \frac{1}{k}\right\}\right)=0 .
$$

Since $\operatorname{dim} K<\infty$, we may assume that $v^{k} \rightarrow v=\left\{v_{n}\right\}_{n \in \mathbb{Z}^{m}}$ in $W$ passing to a subsequence, and thus $\|v\|=1$. By Lemma 2.1 we have $v^{k} \rightarrow v$ in $l^{2}$ and $v_{n}^{k} \rightarrow v_{n}$ for all $n \in \mathbb{Z}^{m}$, so that

$$
\sum_{n \in \mathbb{Z}^{m}}\left|v_{n}^{k}-v_{n}\right|^{2} \rightarrow 0, \quad k \rightarrow \infty
$$

The fact that $\|v\|=1$ implies $\|v\|_{l^{\infty}}=\max _{n \in \mathbb{Z}^{m}}\left|v_{n}\right|>0$. By the definition of norm $\|\cdot\|_{l \infty}$ there exists a constant $\delta_{1}>0$ such that

$$
\sharp\left(\left\{n \in \mathbb{Z}^{m}:\left|v_{n}\right| \geq \delta_{1}\right\}\right) \geq 1 .
$$

Let

$$
\begin{aligned}
& \Omega_{0}=\left\{n \in \mathbb{Z}^{m}|| v_{n} \mid \geq \delta_{1}\right\}, \\
& \Omega_{k}=\left\{n \in \mathbb{Z}^{m}|| v_{n}^{k} \mid<\frac{1}{k}\right\}, \\
& \Omega_{k}^{c}=\mathbb{Z}^{m} \backslash \Omega_{k}=\left\{n \in \mathbb{Z}^{m}|| v_{n}^{k} \mid \geq \frac{1}{k}\right\} .
\end{aligned}
$$

By (A.1) and (A.3) we have

$$
\sharp\left(\Omega_{k} \cap \Omega_{0}\right)=\sharp\left(\Omega_{0} \backslash \Omega_{k}^{c} \cap \Omega_{0}\right) \geq \sharp\left(\Omega_{0}\right)-\sharp\left(\Omega_{k}^{c}\right) \geq 1-0=1 .
$$

Next, we may assume that $\delta_{1}-\frac{1}{k} \geq \frac{1}{2} \delta_{1}$ for $k$ large enough. Then we have

$$
\left|v_{n}^{k}-v_{n}\right|^{2} \geq|| v_{n}^{k}|-| v_{n}||^{2} \geq\left(\delta_{1}-\frac{1}{k}\right)^{2} \geq \frac{1}{4} \delta_{1}^{2}, \quad \forall n \in \Omega_{k} \cap \Omega_{0},
$$

and thus, for all large $k$, we have

$$
\sum_{n \in \mathbb{Z}^{m}}\left|v_{n}^{k}-v_{n}\right|^{2} \geq \sum_{n \in \Omega_{k} \cap \Omega_{0}}\left|v_{n}^{k}-v_{n}\right|^{2} \geq \frac{1}{4} \delta_{1}^{2} \cdot \sharp\left(\Omega_{k} \cap \Omega_{0}\right) \geq \frac{1}{4} \delta_{1}^{2}>0 .
$$

Clearly, it is a contradiction to (A.2). Thus (3.2) holds. 
Availability of data and materials

Not applicable.

\section{Ethics approval and consent to participate}

Not applicable.

\section{Competing interests}

The authors declare that they have no competing interests.

\section{Consent for publication}

Not applicable.

\section{Authors' contributions}

All authors contributed equally to the writing of this paper. All authors read and approved the final manuscript.

\section{Author details}

'School of Mathematical Sciences, University of Jinan, Jinan, Shandong Province 250022, P.R. China. ${ }^{2}$ Sun Yueqi Honors

College, China University Of Mining And Technology, Xuzhou, Jiangsu Province 221116, P.R. China.

\section{Publisher's Note}

Springer Nature remains neutral with regard to jurisdictional claims in published maps and institutional affiliations.

Received: 1 May 2017 Accepted: 29 August 2017 Published online: 15 September 2017

\section{References}

1. Christodoulides, DN, Lederer, F, Silberberg, Y: Discretizing light behaviour in linear and nonlinear waveguide lattices. Nature 424, 817-823 (2003)

2. Kopidakis, G, Aubry, S, Tsironis, GP: Targeted energy transfer through discrete breathers in nonlinear systems. Phys. Rev. Lett. 87, Article ID 165501 (2001). doi:10.1103/PhysRevLett.87.165501

3. Livi, R, Franzosi, R, Oppo, GL: Self-localization of Bose-Einstein condensates in optical lattices via boundary dissipation. Phys. Rev. Lett. 97, Article ID 060401 (2006). doi:10.1103/PhysRevLett.97.060401

4. Chen, G, Ma, S: Discrete nonlinear Schrödinger equations with superlinear nonlinearities. Appl. Math. Comput. 218 5496-5507 (2012)

5. Chen, G, Ma, S: Ground state and geometrically distinct solitons of discrete nonlinear Schrödinger equations with saturable nonlinearities. Stud. Appl. Math. 131, 389-413 (2013)

6. Chen, G, Ma, S: Homoclinic solutions of discrete nonlinear Schrödinger equations with asymptotically or super linear terms. Appl. Math. Comput. 232, 787-798 (2014)

7. Chen, $G, M a, S$, Wang, Z-Q: Standing waves for discrete Schrödinger equations in infinite lattices with saturable nonlinearities. J. Differ. Equ. 261, 3493-3518 (2016)

8. Pankov, A: Gap solitons in periodic discrete nonlinear equations. Nonlinearity 19, 27-40 (2006)

9. Pankov, A: Gap solitons in periodic discrete nonlinear Schrödinger equations. Il. A generalized Nehari manifold approach. Discrete Contin. Dyn. Syst. 19, 419-430 (2007)

10. Pankov, A, Rothos, V: Periodic and decaying solutions in discrete nonlinear Schrödinger with saturable nonlinearity. Proc. R. Soc. A 464, 3219-3236 (2008)

11. Pankov, A: Gap solitons in periodic discrete nonlinear Schrödinger equations with saturable nonlinearities. J. Math. Anal. Appl. 371, 254-265 (2010)

12. Shi, H, Zhang, H: Existence of gap solitons in periodic discrete nonlinear Schrödinger equations. J. Math. Anal. Appl. $361,411-419(2010)$

13. Shi, H: Gap solitons in periodic discrete Schrödinger equations with nonlinearity. Acta Appl. Math. 109, 1065-1075 (2010)

14. Yang, $M$, Chen, $W$, Ding, Y: Solutions for discrete periodic Schrödinger equations with spectrum O. Acta Appl. Math. $110,1475-1488(2010)$

15. Zhou, Z, Yu, J, Chen, Y: On the existence of gap solitons in a periodic discrete nonlinear Schrödinger equation with saturable nonlinearity. Nonlinearity 23, 1727-1740 (2010)

16. Zhou, Z, Yu, J, Chen, Y: Homoclinic solutions in periodic difference equations with saturable nonlinearity. Sci. China Math. 54, 83-93 (2011)

17. Chen, G, Schechter, M: Non-periodic discrete Schrödinger equations: ground state solutions. Z. Angew. Math. Phys. 67, 1-15 (2016)

18. Jia, L, Chen, G: Multiple solutions of discrete Schrödinger equations with growing potentials. Adv. Differ. Equ. 2016, Article ID 275 (2016). doi:10.1186/s13662-016-1003-3

19. Jia, L, Chen, G: Discrete Schrödinger equations with sign-changing nonlinearities: infinitely many homoclinic solutions. J. Math. Anal. Appl. 452, 568-577 (2017)

20. Ma, D, Zhou, Z: Existence and multiplicity results of homoclinic solutions for the DNLS equations with unbounded potentials. Abstr. Appl. Anal. 2012, Article ID 703596 (2012). doi:10.1155/2012/703596

21. Pankov, A, Zhang, G: Standing wave solutions for discrete nonlinear Schrödinger equations with unbounded potentials and saturable nonlinearity. J. Math. Sci. 177, 71-82 (2011)

22. Pankov, A: Standing waves for discrete nonlinear Schrödinger equations: sign-changing nonlinearities. Appl. Anal. 92 , 308-317 (2013)

23. Sun, G: On standing wave solutions for discrete nonlinear Schrödinger equations. Abstr. Appl. Anal. 2013, Article ID 436919 (2013) 
24. Zhang, G, Pankov, A: Standing waves of the discrete nonlinear Schrödinger equations with growing potentials. Commun. Math. Anal. 5, 38-49 (2008)

25. Zhang, G, Liu, F: Existence of breather solutions of the DNLS equations with unbounded potentials. Nonlinear Anal. 71, 786-792 (2009)

26. Zhang, G: Breather solutions of the discrete nonlinear Schrödinger equations with unbounded potentials. J. Math. Phys. 50, Article ID 013505 (2009)

27. Zhang, G, Pankov, A: Standing wave solutions of the discrete non-linear Schrödinger equations with unbounded potentials, II. Appl. Anal. 89, 1541-1557 (2010)

28. Zhou, Z, Ma, D: Multiplicity results of breathers for the discrete nonlinear Schrödinger equations with unbounded potentials. Sci. China Math. 58, 781-790 (2015)

29. Tang, CL, Wu, XP: Periodic solutions for a class of new superquadratic second order Hamiltonian systems. J. Math. Anal. Appl. 34, 65-71 (2014)

30. Rabinowitz, PH: Minimax Methods in Critical Point Theory with Applications to Differential Equations. CBMS Regional Conference Series in Mathematics, vol. 65. Am. Math. Soc., Providence (1986)

31. Willem, M: Minimax theorems. Prog. Nonlinear Differ. Equ. Appl. 50, 139-141 (1996)

\section{Submit your manuscript to a SpringerOpen ${ }^{\circ}$} journal and benefit from:

- Convenient online submission

- Rigorous peer review

- Open access: articles freely available online

- High visibility within the field

- Retaining the copyright to your article

Submit your next manuscript at $\boldsymbol{~ s p r i n g e r o p e n . c o m ~}$ 